\title{
Circular Orbits in Einstein-Gauss-Bonnet Gravity
}

\author{
Valéria M. Rosa* \\ Departamento de Matemática, Universidade Federal de Viçosa, 36570-000 Viçosa, M.G., Brazil \\ Patricio S. Letelier ${ }^{\dagger}$ \\ Departamento de Matemática Aplicada-IMECC, Universidade Estadual de Campinas, 13081-970 Campinas, S.P., Brazil
}

\begin{abstract}
The stability under radial and vertical perturbations of circular orbits associated to particles orbiting a spherically symmetric center of attraction is study in the context of the n-dimensional: Newtonian theory of gravitation, Einstein's general relativity, and Einstein-Gauss-Bonnet theory of gravitation. The presence of a cosmological constant is also considered. We find that this constant as well as the Gauss-Bonnet coupling constant are crucial to have stability for $n>4$.
\end{abstract}

PACS numbers: 04.50.-h

\section{INTRODUCTION}

The physical laws for nonrelativistic phenomena are usually written in a three dimensional space and for relativistic phenomena in a four dimensional spacetime. In both cases these laws admit natural extensions to spaces with arbitrary number of dimensions, these can be taken as the possible existence of an operative principle (or principles) which in additions to these laws lead us to determine the "true dimensions" of the spacetime, the arena where the physical phenomena occur.

Kant observed that the three-dimensionality of the usual space could be related with Newton's inverse square law; " The reason for the three-dimensionality of the space is yet unknown. It is likely that the three-dimensionality of space results from the law according to which the forces on the substances act into each other. The three-dimensionality seems to result from the fact that the substances of the existing work act into each other in such a way that the strength of the effect behaves like the inverse of the square of the distances" [1] 2].

Since in a first approximation, the galaxies and planetary systems can be considered as formed by particles moving in circular orbits around an attraction center, a possible principle to determine the dimensionality of the space is the existence of stable circular orbits. The study of the stability of circular orbits moving in potentials solutions of the n-dimensional Laplace's equations can be found in [3]. These studies on stability culminate with the Ehrenfest work [4] where he shows that in the Newtonian-Keplerian problem there exists stable limited orbit if and only if the dimension of the space is three (the potential must be null at infinity).

In Einstein's gravity Tangherlini [5] study the stability under radial perturbation of orbits associated to test particles moving around a $n$-dimensional Schwarzschild black hole. He found stability for $n=4$ only, i.e., in complete concordance with the Newtonian case. The dimension of the constant time slice, $n-1$, will be denoted by $d$.

Generalization of the Newton theory that consists in the addition of a cosmological constant was studied by several authors, see for instance [6] (cosmological context) and 7] (potential theory context). Clearly this generalization is motivated by the success of Einstein's gravity with cosmological constant. An example of model that makes use of a cosmological constant is the $\Lambda$ Cold Dark Matter model $(\Lambda \mathrm{CDM})$ [8] that explains in a simple way the observed acceleration of the Universe as well as the cosmic microwave background.

The Hilbert Lagrangian (Ricci scalar times square root of the metric determinant) is a divergence in two dimensions. We have that the Hilbert action is proportional to the Euler characteristic of the two dimensional manifold (GaussBonnet theorem [9]). In four dimensions we have that the Bach-Lanczos Lagrangian [10] 11] (a particular combination of terms quadratic in the Riemann-Christoffel curvature tensor) is a divergence, so in four dimensions this Lagrangian has no dynamical significance, like the Hilbert Lagrangian in two dimensions. The simplest generalization of the Hilbert Lagrangian for $n$-dimensional spaces $(n>4)$ can be obtained adding the Bach-Lanczos Lagrangian to the Hilbert one. This new theory of gravitation, Einstein-Gauss-Bonnet (EGB) theory, has some remarkable mathematical properties, see for instance [12].

Another outstanding feature of the Einstein-Gauss-Bonnet theory is that the Bach-Lanczos action appears as the leading quadratic correction term of the Hilbert action in the expansion of the supersymmetric string theory [13]. To be more precise the Bach-Lanczos action appears exactly in the expansion of the $E_{8} \times E_{8}$ heterotic string model [14].

\footnotetext{
* e-mail: vmrosa@ufv.br

$\dagger$ e-mail: letelier@ime.unicamp.br
} 
The aim of this paper is to study the stability under radial perturbations as well as vertical perturbations of circular orbits related to test bodies orbiting a spherically symmetric center of attraction in the above mentioned theories of gravity in an arbitrary number of dimensions.

In Section II we study the stability of circular orbits in a $n$-dimensional Newtonian context with cosmological constant. In Section III we consider the stability of circular orbits in the EGB theory. We divide the study of stability in two. First, in Sect. IV, we consider the case without cosmological constant. In the next section (Sect. V) we analyze the case where this constant is not null. We end summarizing our results in Sect. V.

\section{II. $d$-DIMENSIONAL NEWTONIAN CENTER OF ATTRACTION WITH COSMOLOGICAL CONSTANT}

The Laplace equation with cosmological constant is

$$
\nabla^{2} \Phi+\Lambda=0
$$

where $\nabla^{2}$ is the Laplacian operator in $d$-dimensions, $\Phi$ and $\Lambda$ are the Newtonian potential and the cosmological constant, respectively. For spherical symmetry Eq. (1) reduces to

$$
\frac{1}{r^{n-2}} \frac{\partial}{\partial r}\left(r^{n-2} \frac{\partial}{\partial r} \Phi\right)+\Lambda=0
$$

that has as solution for $n \geq 4$,

$$
\Phi=-\frac{C}{n-3} \frac{1}{r^{n-3}}-\frac{\Lambda}{2(n-1)} r^{2}
$$

where $C$ is an integration constant, that for $n=4(d=3)$ it is proportional to the mass of the center of attraction $(C=G M$, with $G$ the Newton's constant of gravitation).

The Newton equations for an axially symmetric potential can be written as

$$
\begin{aligned}
& \ddot{R}-R \dot{\varphi}^{2}=-\frac{\partial \Phi}{\partial R}, \\
& \frac{d}{d t}\left(R^{2} \dot{\varphi}\right)=0, \\
& \ddot{z^{i}}=-\frac{\partial \Phi}{\partial z^{i}},
\end{aligned}
$$

where the overdots denote derivation with respect to $t,\left(R, \varphi, z^{1}, \ldots, z^{d-2}\right)$ are $d$-dimensional cylindrical coordinates, and $\Phi=\Phi\left(R, z^{1}, \ldots, z^{d-2}\right)$. Defining the effective potential $\Phi_{e f f}=\Phi+h^{2} /\left(2 R^{2}\right)$, with $h=R^{2} \dot{\varphi}(=\mathrm{constant})$, we find,

$$
\ddot{R}=-\frac{\partial \Phi_{e f f}}{\partial R}, \ddot{z^{i}}=-\frac{\partial \Phi_{e f f}}{\partial z^{i}}
$$

Now let us study the stability of the circular orbit $R=R_{0}, z^{i}=0$. We shall assume that the potential has reflection symmetry on the planes $z^{i}=0$, i.e., $\Phi=\Phi\left[R,\left(z^{1}\right)^{2}, \ldots,\left(z^{d-2}\right)^{2}\right]$. Expanding the effective potential around this circular orbit we find,

$$
\Phi_{e f f}=\Phi_{e f f}\left(R_{0}, 0, \ldots, 0\right)+\frac{1}{2} \frac{\partial^{2} \Phi_{e f f}\left(R_{0}, 0, \ldots, 0\right)}{\partial R^{2}}\left(R-R_{0}\right)^{2}+\frac{1}{2} \sum_{i=1}^{d-2} \frac{\partial^{2} \Phi_{e f f}\left(R_{0}, 0, \ldots, 0\right)}{\partial\left(z^{i}\right)^{2}}\left(z^{i}\right)^{2}+\cdots
$$

From Newton's equations we find the motion for the perturbations $\rho=R-R_{0}$ and $\zeta^{i}=z^{i}-0$,

$$
\ddot{\rho}+\kappa^{2} \rho=0, \ddot{\zeta}^{i}+\nu_{i}^{2} \zeta^{i}=0,
$$

where

$$
\begin{aligned}
& k^{2}=\frac{\partial^{2} \Phi_{e f f}\left(R_{0}, 0, \ldots, 0\right)}{\partial R^{2}}=\frac{\partial^{2} \Phi\left(R_{0}, 0, \ldots, 0\right)}{\partial R^{2}}+\frac{3}{R} \frac{\partial \Phi\left(R_{0}, 0, \ldots, 0\right)}{\partial R}, \\
& \nu_{i}^{2}=\frac{\partial^{2} \Phi_{e f f}\left(R_{0}, 0, \ldots, 0\right)}{\partial\left(z^{i}\right)^{2}}=\frac{\partial^{2} \Phi\left(R_{0}, 0, \ldots, 0\right)}{\partial\left(z^{i}\right)^{2}}
\end{aligned}
$$


Thus when $\kappa^{2}>0$ we have that the radial perturbation remains bounded, i.e., we have linear stability. This perturbations are usually named as epicyclic perturbations [15]. In a similar way when $\nu_{i}^{2}>0$ we have that the "vertical" perturbation $\zeta^{i}$ also remains bounded. In this case we say that we have linear stability under perturbations along the direction $z^{i}$. For the spherically symmetric potential (3) we find

$$
\begin{aligned}
& \kappa^{2}=(5-n) C R_{0}^{1-n}-4 \Lambda /(n-1), \\
& \nu_{i}^{2}=\nu^{2}=C R_{0}^{1-n}-\Lambda /(n-1) .
\end{aligned}
$$

To derive the previous equations we used $r=\left[R^{2}+\sum_{i=1}^{d-2}\left(z^{i}\right)^{2}\right]^{\frac{1}{2}}$. Note that because of the spherical symmetry of the potential we have that all the frequencies for all the vertical perturbations are equal.

The relations (12) and (13) tell us that when $\Lambda=0$ we have stability only for $n=4$. For $\Lambda>0$ and $n=4$ we have stability whenever $R_{0}^{3}<3 C /(4 \Lambda)$. These last relation puts an upper limit to the size of the stable galactic structures. For $\Lambda>0$ and $n \geq 5$ we have $\kappa^{2}<0$ and the circular orbits are not stable under epicyclic perturbations. Now for $\Lambda<0$ we have stable circular orbits for $n=4,5$ and for $n \geq 6$ we have stability for $R_{0}$ limited by the lower bound $R_{0}^{*} \equiv[(n-5)(n-1) C /(-4 \Lambda)]^{1 /(n-1)}\left(<R_{0}\right)$. In other words for certain values of $n$ the presence of a cosmological constant can stabilize the circular orbits, sometimes with limitations in the size of the orbit.

\section{EINSTEIN-GAUSS-BONNET THEORY OF GRAVITATION}

The Einstein-Gauss-Bonnet action is,

$$
S=\int d^{n} x \sqrt{-g}\left[\frac{1}{\kappa_{n}^{2}}\left(R-2 \Lambda+\alpha L_{G B}\right)\right]+S_{\text {matter }}
$$

where $R$ is the $n$-dimensional Ricci scalar $(n>4), \kappa_{n}$ is defined as $\kappa_{n} \equiv \sqrt{8 \pi G_{n}}$, where $G_{n}$ is the $n$-dimensional gravitational constant. We use units such that the speed of light is one $(c=1)$. The Gauss-Bonnet term $L_{G B}$ is given by

$$
L_{G B}=R^{2}-4 R_{\mu \nu} R^{\mu \nu}+R_{\mu \nu \rho \delta} R^{\mu \nu \rho \delta}
$$

and $\alpha$ is a coupling constant. $S_{\text {matter }}$ is the part of the action that describes the matter. We use the conventions, $R_{b c d}^{a}=\partial_{c} \Gamma_{b d}^{a}+\cdots, R_{\mu \nu}=R_{\mu \rho \nu}^{\rho}$ and $R=R_{\mu}^{\mu}$. The action (14) is the simplest one for $n>4$ built with topological terms. For the general case see [16].

From (14) we find

$$
G_{\mu \nu}-\alpha H_{\mu \nu}-\Lambda g_{\mu \nu}=\kappa_{n}^{2} T_{\mu \nu}
$$

where

$$
\begin{aligned}
G_{\mu \nu} & =R_{\mu \nu}-\frac{1}{2} g_{\mu \nu} R \\
H_{\mu \nu} & =2\left[R R_{\mu \nu}-2 R_{\mu \alpha} R_{\nu}^{\alpha}-2 R^{\alpha \beta} R_{\mu \alpha \nu \beta}+R_{\mu}^{\alpha \beta \gamma} R_{\nu \alpha \beta \gamma}\right]-\frac{1}{2} g_{\mu \nu} L_{G B} .
\end{aligned}
$$

For vacuum $\left(T_{\mu \nu}=0\right)$ spherically symmetric solutions to the EGB equations there is not lost of generality in choosing the metric,

$$
d s^{2}=f(r) d t^{2}-f(r)^{-1} d r^{2}-r^{2} d \Omega_{n-2}^{2},
$$

where $d \Omega_{n-2}^{2}=d \theta_{2}^{2}+\Sigma_{j=3}^{n}\left(\Pi_{i=2}^{n-1} \sin ^{2} \theta_{i}\right) d \theta_{j}^{2}$ is the metric of a $(n-2)$-sphere. The case in which this sphere is changed by a space of negative or null curvature has also been considered [18].

From the fact that the the Einstein tensor, $G^{\mu \nu}$, as well as the Gauss Bonnet tensor, $H^{\mu \nu}$, are divergence free [16] we have that equation (16) tells us that $T^{\mu \nu}$ is also divergence free. From this last fact we conclude that test particles move along geodesics [17]. We shall analyze the stability of the circular geodesics $\gamma$ whose parametric equation is,

$$
t=t(s), \quad r=r_{0}, \theta_{j}=\frac{\pi}{2}, j=2, \ldots, n-1, \quad \theta_{n}=\phi \in[0,2 \pi] .
$$

The evolution of a small perturbation, $\xi^{\mu}$, of $\gamma$ is given by the geodesic deviation equation,

$$
\frac{D^{2} \xi^{\mu}}{d s^{2}}+R_{\nu \alpha \beta}^{\mu} u^{\nu} \xi^{\alpha} u^{\beta}=0
$$


where $D / d s=u^{\mu} \nabla_{\mu}$, and $u^{\mu}=d x^{\mu} / d s$. Equation (20) can be cast in a simpler, but not manifestly covariant way, as

$$
\ddot{\xi}^{\mu}+2 \Gamma_{\alpha \beta}^{\mu} u^{\alpha} \dot{\xi}^{\beta}+\Gamma_{\alpha \beta, \nu}^{\mu} u^{\alpha} u^{\beta} \xi^{\nu}=0,
$$

where the comma denotes ordinary differentiation. For the curve (19) the system (21) reduces to,

$$
\begin{aligned}
& \ddot{\xi}^{0}+\frac{f^{\prime}\left(r_{0}\right)}{f\left(r_{0}\right)} \dot{t} \dot{\xi}^{1}=0, \\
& \ddot{\xi}^{1}+\kappa^{2} \xi^{1}=0, \\
& \ddot{\xi}^{j}+\nu^{2} \xi^{j}=0, \\
& \ddot{\xi}^{n}+\frac{2}{r_{0}} \dot{\phi} \dot{\xi}^{1}=0,
\end{aligned}
$$

where

$$
\begin{aligned}
\kappa^{2} & =\frac{f\left(r_{0}\right)\left(r_{0} f^{\prime \prime}\left(r_{0}\right)+3 f^{\prime}\left(r_{0}\right)\right)-2 r_{0} f^{\prime}\left(r_{0}\right)^{2}}{r_{0}\left(2 f\left(r_{0}\right)-r_{0} f^{\prime}\left(r_{0}\right)\right)}, \\
\nu^{2} & =\frac{f^{\prime}\left(r_{0}\right)}{r_{0}\left(2 f\left(r_{0}\right)-r_{0} f^{\prime}\left(r_{0}\right)\right)} .
\end{aligned}
$$

We shall do a study of cases depending on the values of $\alpha$ and $\Lambda$. The analysis starts with the stability of circular orbits for the pure Einsteinian case (no Gauss-Bonnet term). Then we study the stability of these orbits when a coupling term and/or the cosmological constant are considered.

For $\Lambda=0$ and $\alpha=0$, the model of spacetime reduces to the $n$-dimensional Schwarzschild solution. The equation for the metric function is

$$
r f^{\prime}-(n-3)(1-f)=0,
$$

which has the general solution $f(r)=1-C / r^{n-3}$. $C$ is an integration constant that will taken as positive in order to have the Schwarzschild solution when $n=4(C=2 G M)$. In this case the constants $\nu^{2}$ and $\kappa^{2}$ can be cast as,

$$
\begin{aligned}
\nu^{2} & =\frac{C(n-3) r_{0}^{n-3}}{r_{0}^{n-1}\left(2 r_{0}^{n-3}-C(n-1)\right)}, \\
\kappa^{2} & =\frac{C(n-3)\left((5-n) r_{0}^{n-3}-C(n-1)\right)}{r_{0}^{n-1}\left(2 r_{0}^{n-3}-C(n-1)\right)} .
\end{aligned}
$$

Therefore, we have $\nu^{2}>0$ if and only if $r_{0}^{n-3}>C(n-1)$, this imply that $\kappa^{2}>0$ if and only if $(5-n) r_{0}^{n-3}-C(n-1)>$ 0 , and it only occurs when $n=4$ and $r_{0}>6 G M$. Hence, we have stable circular orbits only in a 4 -dimensional spacetime as it was expected.

\section{EINSTEIN-GAUSS-BONNET THEORY WITH $\Lambda=0$.}

Taking $\Lambda=0$ in (16) with $T_{\mu \nu}=0$, the equation of metric function $f$ is

$$
r f^{\prime}-(n-3)(1-f)\left[-1+\frac{\alpha(n-4)}{r^{2}}\left(2 r f^{\prime}-(n-5)(1-f)\right)\right]=0,
$$

which has the general solution

$$
f_{ \pm}(r)=1+\frac{r^{2}}{2 \tilde{\alpha}}\left[1 \pm \sqrt{1+\frac{4 \tilde{\alpha} C}{r^{n-1}}}\right]
$$

where $\tilde{\alpha}=(n-3)(n-4) \alpha$ and $C$ is an integration constant. The sign of $C$ is chosen analyzing the behavior of $f$ for $|\alpha| \rightarrow 0$. Let $f_{+}$be the function $f$ where the sign of square root is positive and let $f_{-}$be the other case. When $\alpha$ is small, the asymptotic behavior of $f_{-}$is given by

$$
f_{-} \approx 1-\frac{C}{r^{n-3}} .
$$



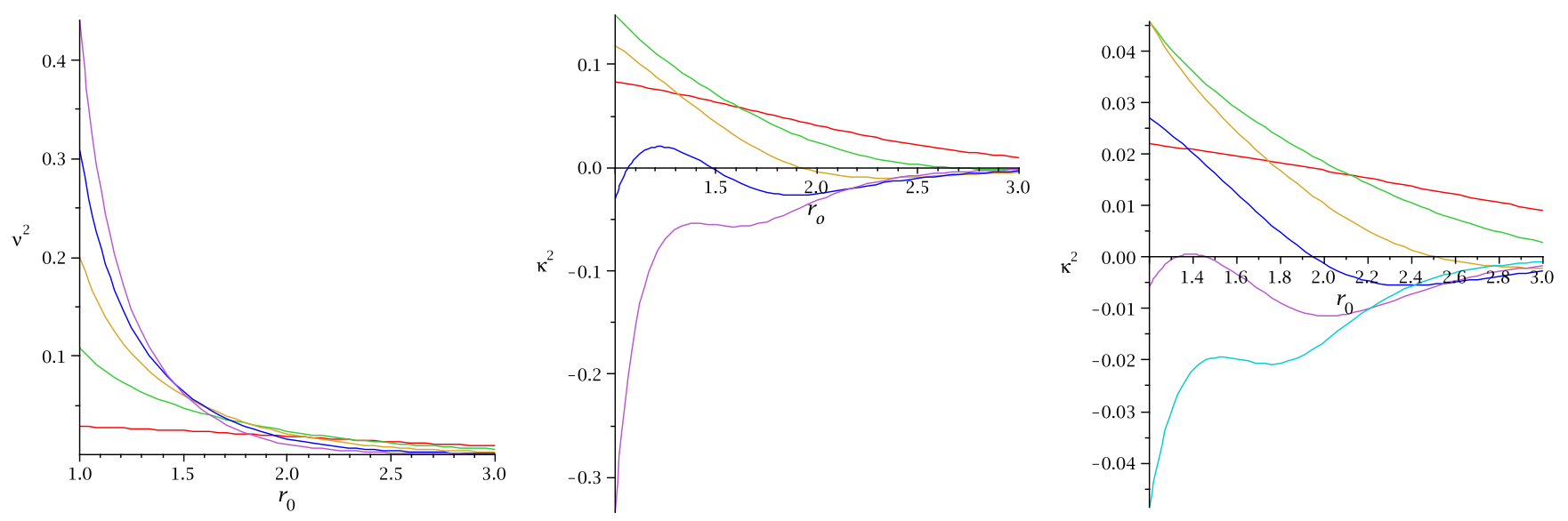

FIG. 1: GR branch, $\Lambda=0$ case. Epicyclic and vertical frequencies for several values of $n$ and $C=1,1 \leq r_{0} \leq 3$. For the first two figures $\tilde{\alpha}=20$ and for the third $\tilde{\alpha}=80$. In the first graphic $n$ take the values $n=9$ (top curve), 8,7,6 and 5 (bottom curve). In the second, counting along the line $r_{0}=2, n$ takes the values 5 (top curve), 6, 7, 8 and 9 (bottom curve). And for the third graphic, counting along the same line, $n=6$ (top line), 5, 7,8,9, 10 (bottom line). We see that the region of stability shrinks and disappears for large $n$.

So, considering the Gauss-Bonnet term as a perturbation of the $n$-dimensional Schwarzschild solution, $C$ must be a positive constant.

When $\alpha$ is small, the asymptotic behavior of $f_{+}$is given by

$$
f_{+} \approx 1+\frac{r^{2}}{\tilde{\alpha}}+\frac{C}{r^{n-3}} .
$$

For $\alpha>0$, it corresponds to Schwarzschild-anti-de Sitter spacetime with negative gravitational mass, with the standard energy definition in background anti-de Sitter [19].

Then, there are two families of solutions corresponding to the sign in front of the square root that appears in (29). Following the definition given in 20], the family with minus (plus) sign will be called general relativity (GR) branch (non-GR branch) solution. The spacetime structure of these solutions of the EGB theory is studies in [21].

\section{A. The GR branch solution.}

By chosing the solution $f=f_{-}$of (28) from (26) we find,

$$
\begin{aligned}
\nu^{2} & =\frac{r_{0}^{n}(1-\sqrt{\beta})+C r_{0} \tilde{\alpha}(5-n)}{\tilde{\alpha}\left(r_{0}^{3} C(n-1)-2 r_{0}^{n} \sqrt{\beta}\right)}, \\
\kappa^{2} & =\frac{A_{1}+A_{2}+A_{3}}{-2 r_{0}^{n} \beta \sqrt{\beta} \tilde{\alpha}\left(-2 r_{0}^{n} \sqrt{\beta}+r_{0}^{3} C(n-1)\right)},
\end{aligned}
$$

where $\beta=1+4 \tilde{\alpha} C r_{0}^{1-n}$ and

$$
\begin{aligned}
& A_{1}=8 r_{0}^{2 n}(1-\sqrt{\beta})+r_{0}^{n+3} C\left(n^{2}-1\right)(1-\sqrt{\beta}), \\
& A_{2}=2 r_{0}^{n+1}\left[32-\sqrt{\beta}\left(n^{2}-8 n+39\right)\right]+2 r_{0}^{4} C^{2} \tilde{\alpha}(n-1)[n+7-\sqrt{\beta}(n+3)], \\
& A_{3}=4 r_{0}^{2} C^{2} \tilde{\alpha}^{2}[32-2 \sqrt{\beta} \tilde{\alpha} C(n-5)(n-9)]-8 r_{0}^{5-n} C \tilde{\alpha}(n-5)(n-1) .
\end{aligned}
$$

The behavior of the functions $\nu^{2}$ and $\kappa^{2}$ are not simple, but it is possible to find $n, \tilde{\alpha}, C$ and $r_{0}$ such that $\nu^{2}$ and $\kappa^{2}$ are both positive, i.e., there are stable circular orbits. For example, we have stable circular orbits when $n=5$, $\tilde{\alpha}=2, C=1$ and $0.2 \leq r_{0} \leq 2.0 ; n=6, \tilde{\alpha}=20, C=1$ and $0.2 \leq r_{0} \leq 2.0 ; n=7, \tilde{\alpha}=20, C=1$ and $0.7 \leq r_{0} \leq 1.9$; $n=8, \tilde{\alpha}=20, C=1$ and $1.07 \leq r_{0} \leq 1.47$; and $n=9, \tilde{\alpha}=80, C=1$ and $1.33 \leq r_{0} \leq 1.46$. In all these examples we have $f_{-}\left(r_{0}\right)>0$ in the specified region. Hence, the Gauss-Bonnet term can stabilize circular orbits in some higher dimensions spacetimes. 

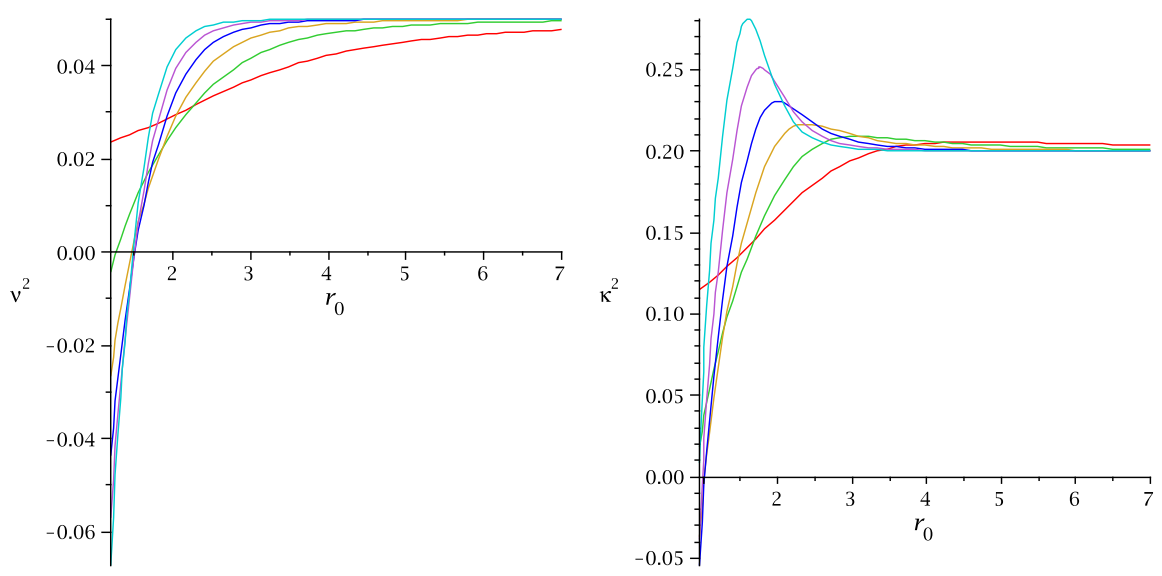

FIG. 2: Non-GR branch, $\Lambda=0$ case. Graphics of the functions $\nu^{2}$ and $\kappa^{2}$ for $\tilde{\alpha}=20, C=1$ and $1 \leq r_{0} \leq 7$ and $n=10$ (top line), $9,8,7,6,5$ (bottom line). The have a large region of stability.

In Fig. 1 we show the epicyclic and vertical frequencies for several values of $n$ and $C=1,1 \leq r_{0} \leq 3$. For the first two figures $\tilde{\alpha}=20$ and for the third $\tilde{\alpha}=80$. In the first graphic $n$ take the values $n=9$ (top curve), $8,7,6$ and 5 (bottom curve). In the second graphic, counting along the line $r_{0}=2, n$ takes the values 5 (top curve), 6,7,8 and 9 (bottom curve). There is no stable circular orbit when $n=9$. And for the third graphic, counting along the same line, $n=6$ (top line), 5, 7,8,9,10 (bottom line). In this case here is no stable circular orbit when $n=10$, but for $n=9$ we have stability.

The vertical frequency, $\nu^{2}$, is always positive no matter the value of $n$ but the range of $r_{0}$ where $\kappa^{2}$ is positive decreases when the dimension $n$ increases.

For $n \geq 10$ and $\tilde{\alpha}>0$, and also for $n \geq 5$ and $\tilde{\alpha}<0$, we calculate the functions $\nu^{2}$ and $\kappa^{2}$ into a grid of points with $0.1 \leq r_{0} \leq 2$ (step 0.1), $5 \leq n \leq 20$ and $0.01 \leq \tilde{\alpha} \leq 100$ (step 0.01 into [0.01, 0.1], step 0.1 into [0.1, 1], step 1 into $[1,100])$ and $C=1$. In the case $\alpha<0$ we work with $-100 \leq \tilde{\alpha} \leq-0.01$, with the same steps for the corresponding intervals. In all these numerical examples we obtain $\nu^{2} \kappa^{2}<0$ and, therefore, no circular orbit is stable.

\section{B. The non-GR branch solution.}

For completeness we study the case when $f=f_{+}$is the solution of (28). In this case the constants $\nu^{2}$ and $\kappa^{2}$ are given as in (32) and (33) replacing $-\sqrt{\beta}$ by $\sqrt{\beta}$.

Also in this case, the stability depends on the sign of $\alpha$. When $\alpha<0$, in the expression for $\nu^{2}=N_{\nu} / D_{\nu}$, we have a numerator, $N_{\nu}=r_{0}^{n}\left[(1+\sqrt{\beta}) r_{0}^{n}-(n-5) C \tilde{\alpha} r_{0}\right]$, non negative and a denominator, $D_{\nu}=\tilde{\alpha}\left(r_{0}^{3} C(n-1)+2 r_{0}^{n} \sqrt{\beta}\right)$, always negative. So, $\nu^{2}$ is not positive, hence there are no stable circular orbits.

The parameters $n(\geq 5)$ and $C$ are positive. When $\alpha>0$ the denominators of $\nu^{2}$ and $\kappa^{2}$ are always positive $\left(D_{\kappa}=2 \beta \sqrt{\beta} r_{0}^{n} D_{\nu}\right)$. The same happens with the functions $f_{+}, \beta$, and with the coefficients of $r_{0}$ in $\bar{A}_{1}$ and $\overline{A_{2}}$ (Note that $\bar{A}_{i}$ is the function $A_{i}$ after replacing $-\sqrt{\beta}$ by $\left.\sqrt{\beta}, i=1,2,3\right)$. These positive coefficients assure that, for $r_{0}$ sufficiently large, the numerator of $\nu^{2}, N_{\nu}=\left[(1+\sqrt{\beta}) r_{0}^{n}-(n-5) C \tilde{\alpha} r_{0}\right]$, and the numerator of $\kappa^{2}, N_{\kappa}=\left[\bar{A}_{1}+\bar{A}_{2}+\bar{A}_{3}\right]$, are positive. Hence, we conclude that it is possible to find stable circular orbits for $n \geq 5$ and $\alpha>0$.

Fig. 2 shows the functions $\nu^{2}$ and $\kappa^{2}$, for $C=1, \tilde{\alpha}=20,1 \leq r \leq 7$ and $n=10$ (top line), 9, 8, 7, 6, 5 (bottom line). We have stability for $r_{0}>r_{n}$, where $r_{n}$ depends on the dimension $n$.

\section{EINSTEIN-GAUSS-BONNET THEORY WITH $\Lambda \neq 0$.}

Taking $\alpha=0$ and $\Lambda \neq 0$ in (16) with $T_{\mu \nu}=0$, the metric function obeys the equation,

$$
r f^{\prime}-(n-3)(1-f)+\frac{2 \Lambda r^{2}}{n-2}=0,
$$


which has the general solution,

$$
f(r)=1-\frac{C}{r^{n-3}}-\frac{2 \Lambda r^{2}}{(n-1)(n-2)}
$$

the integration constant $C$ will be considered positive as before. The frequencies (26) in this case are $\nu^{2}=N_{1} / D_{1}$ and $\kappa^{2}=N_{2} / D_{2}$, where

$$
\begin{array}{r}
N_{1}=C r_{0}^{2}(n-1)(n-2)(n-3)^{2}-4 \Lambda r_{0}^{n+1} ; \quad D_{1}=r_{0}^{4}(n-1)(n-2)\left(2 r_{0}^{n-3}-C(n-1)\right), \\
N_{2}=C r_{0}^{2}(n-2)(n-3)^{2}\left((5-n) r_{0}^{n-3}-C(n-1)\right)-2 \Lambda r_{0}^{n+1}\left(8 r_{0}^{n-3}-C\left(n^{2}-1\right)\right), \\
D_{2}=r_{0}^{n+1}\left(2 r_{0}^{n-3}-C(n-1)\right)(n-1)(n-2) .
\end{array}
$$

When $\Lambda<0$, the numerator $N_{1}$ of $\nu^{2}$ is positive for all $r_{0}$, and its denominator $D_{1}$ is positive if and only if $r_{0}^{n-3}>C(n-1) / 2$. Note that $D_{2}$ change of sign with $D_{1}$. The function $f$ is positive when $r_{0}^{n-3}>C$, and this condition is satisfied when $D_{1}>0$. So, it is necessary to find the values of $r_{0}$ for which the numerator $N_{2}$ of $\kappa^{2}$ is positive. For better visualization, we write $\Lambda=-\lambda$ in $N_{2}$

$$
N_{2}=16 \lambda r_{0}^{2 n-2}-2 C \lambda\left(n^{2}-1\right) r_{0}^{n+1}-C(n-2)(n-3)(n-5) r_{0}^{n-1}-C^{2}(n-2)(n-3)(n-1) r_{0}^{2} .
$$

Analyzing the variation of the signs of the polynomial $N_{2}$, we can see that there is at least one positive root for $N_{2}$. Adding the fact that for large $r_{0}, N_{2}$ is positive, we can conclude that it is possible to find a value $\bar{r}$ such that $\nu^{2}>0$ and $\kappa^{2}>0$ for $r_{0}>\bar{r}$. Therefore, when $\Lambda<0$ and for $n \geq 4$ there exist stable circular orbits.

When $\Lambda>0$, in order to have $f>0$ it is necessary that, at least,

$$
2 r^{n-3}-C(n-1)>0 .
$$

Under this condition the denominators $D_{1}$ and $D_{2}$ are positive. Now it is necessary that the numerators $N_{1}$ and $N_{2}$ be also positive. The polynomial $N_{1}$ is positive when

$$
C r_{0}^{2}(n-1)(n-2)(n-3)^{2}-4 \Lambda r_{0}^{n+1}>0 .
$$

In four dimensions we have $N_{1}>0$ and $N_{2}>0$ when we take, for example, $C=0.1, \Lambda=0.05$ and $0.32 \leq r_{0} \leq 0.55$. But when $n \geq 5$ the numerators, $N_{1}$ and $N_{2}$, can not be positive at same time. We can see this by doing an analysis of the polynomial $N_{2}$. Using (37) and (38) we obtain

$$
\begin{aligned}
& N_{2}<-2 \Lambda r_{0}^{n+1}\left(4 C(n-1)-C\left(n^{2}-1\right)\right)-C r_{0}^{2}(n-2)(n-3)^{2}((5-n) C(n-1) / 2-C(n-1))= \\
& =C(n-1)(n-3)\left[4 \Lambda r_{0}^{n+1}-C r_{0}^{2}(n-1)(n-2)(n-3)^{2}\right] / 2<0 .
\end{aligned}
$$

So, for $n \geq 5$ and $\Lambda>0$ there is no stable circular orbit. It is interesting to note that the addition of a negative cosmological constant stabilize, former not stable, circular orbits for any $n \geq 4$.

In the following we analyze the stability of the circular orbits when both $\Lambda$ and $\alpha$ are not null. In this case the equation of metric function is written as

$$
r f^{\prime}-(n-3)(1-f)+\frac{2 \Lambda r^{2}}{n-2}+\frac{\alpha(n-4)(n-3)(1-f)}{r^{2}}\left(2 r f^{\prime}-(n-5)(1-f)\right)=0,
$$

which has the general solution

$$
f(r) \pm=1+\frac{r^{2}}{2 \tilde{\alpha}}\left[1 \pm \sqrt{1+\frac{4 \tilde{\alpha}}{(n-1)(n-2)}\left(2 \Lambda+\frac{C}{r^{n-1}}\right)}\right]
$$

where $\tilde{\alpha}=(n-3)(n-4) \alpha$ and $C$ is a integration constant. As before, let $f_{+}$be the function $f$ when the sign of square root is positive and let $f_{-}$be the other case. In the limit $|\alpha| \rightarrow 0$ the function $f_{-}$reduce to

$$
f_{-} \approx 1-\frac{2 \Lambda r^{2}}{(n-1)(n-2)}-\frac{C}{(n-1)(n-2) r^{n-3}} .
$$

By considering the Gauss-Bonnet term as a perturbation of the Schwarzschild-dS (Schwarzschild-adS) spacetime with $\Lambda>0(\Lambda<0)$, we conclude that $C$ must be positive. 
When $\alpha$ is small, the asymptotic behavior of $f_{+}$is given by

$$
f_{+} \approx 1+\frac{r^{2}}{\tilde{\alpha}}+\frac{2 \Lambda r^{2}}{(n-1)(n-2)}+\frac{C}{(n-1)(n-2) r^{n-3}} .
$$

As we can see, here again, there are two families of solutions corresponding to the sign in front of the square root in (41). We define two branches following the same criterion, the GR branch and the non-GR branch.

\section{A. The GR branch solution $(\Lambda \neq 0)$}

The functions $\nu^{2}$ and $\kappa^{2}$ in this case can be written as (26)

$$
\begin{aligned}
\nu^{2} & =\frac{r_{0}^{n}\left[(n-1)(n-2)\left(1-\sqrt{\beta_{*}}\right)+8 \Lambda \tilde{\alpha}-\tilde{\alpha} C r_{0}^{1-n}(n-5)\right]}{\tilde{\alpha}(n-1)\left(-2 r_{0}^{n} \sqrt{\beta_{*}}(n-2)+r_{0}^{3} C\right)}, \\
\kappa^{2} & =\frac{B_{4} r_{0}^{n+3}+B_{3} r_{0}^{n+1}+B_{2} r_{0}^{n}+B_{1} r_{0}^{4}+B_{0} r_{0}^{2}}{2 \tilde{\alpha}(n-1)\left(-2 r_{0}^{n} \sqrt{\beta_{*}}(n-2)+r_{0}^{3} C\right) r_{0}^{n}\left[(n-1)(n-2)+4 \tilde{\alpha}\left(2 \Lambda+C r_{0}^{1-n}\right)\right]}
\end{aligned}
$$

where

$$
\beta_{*}=1+\frac{4 \tilde{\alpha}}{(n-1)(n-2)}\left(2 \Lambda+\frac{C}{r^{n-1}}\right)
$$

and

$$
\begin{aligned}
& B_{4}=C(8 \Lambda \tilde{\alpha}+(n-1)(n-2))\left(n^{2}-1\right)\left[-\sqrt{\beta_{*}}+1\right], \\
& B_{3}=C\left[-32 \tilde{\alpha} \sqrt{\beta_{*}}(n-1)(n-2)+2 \tilde{\alpha}\left(n^{2}-8 n+39\right)(8 \tilde{\alpha} \Lambda+(n-1)(n-2))\right], \\
& B_{2}=8\left[\left[\left(-\sqrt{\beta_{*}}+1\right)(n-1)(n-2)+8 \tilde{\alpha} \Lambda\right][(n-1)(n-2)+8 \tilde{\alpha} \Lambda]\right], \\
& B_{1}=2 C^{2} \tilde{\alpha}(n-1)\left[-\sqrt{\beta_{*}}(5-n)+n+3\right], \\
& B_{0}=4 \tilde{\alpha}^{2} C^{2}[(n-5)(n-9)] .
\end{aligned}
$$

First, when $\Lambda<0$ it is possible to find stable circular orbits. For example, if $\tilde{\alpha}= \pm 1, \Lambda=-1, C=1$ then $\gamma$ is stable when $r_{0} \geq 1.2(n \geq 5)$. Note that $f_{-}=0$ if and only if

$$
p(r)=2(-\Lambda) r^{n+4}+(n-1)(n-2) r^{n+2}+(n-1)(n-2) \tilde{\alpha} r^{n}-C r^{5}=0 .
$$

Analyzing the variation of signs in the coefficients of $p$, we can see that, if $\Lambda<0$ then the number of variations is one and there is only one positive value $\bar{r}$ such that $p(\bar{r})=0$. And for $r_{0}>\bar{r}$ we have $f\left(r_{0}\right)>0$. If $\Lambda>0$, the existence of a root of $p(r)=0$ depends on the relation between $\tilde{\alpha}, \Lambda$ and $\mathrm{C}$. In the cases $\tilde{\alpha}= \pm 1, \Lambda=-1, C=1$, the polynomial $p(r)=2 r^{n+4}+(n-1)(n-2)\left(r^{2}-1\right) r^{n}-r^{5}$ has only one positive root. Hence, we have a horizon $\left(\right.$ at $\left.r_{H}<1\right)$ with the zone of stability outside this horizon.

Figure 3 shows the behavior of the functions $\nu^{2}$ and $\kappa^{2}$ for $\Lambda=-1, C=1, \tilde{\alpha}=1$ and $1 \leq r_{0} \leq 3$, and $n=5($ top curve), 6,7,8,9,10 (bottom curve). We see a small region of instability for large $n$. Similar behavior is found for the same parameters, but $\tilde{\alpha}=-1$.

The cases where $\Lambda>0$ was numerically analyzed. For example, we have stable circular orbits when $n=5$, $\tilde{\alpha}=C=\Lambda=1$ and $0.1 \leq r_{0} \leq 0.5 ; n=6, \tilde{\alpha}=C=\Lambda=1$ and $0.2 \leq r_{0} \leq 0.55 ; n=7, \tilde{\alpha}=2, C=\Lambda=1$ and $0.3 \leq r_{0} \leq 0.55 ; n=\overline{8}, \tilde{\alpha}=9, C=\Lambda=1$ and $0.45 \leq r_{0} \leq 0.55$, and $n=9, \tilde{\alpha}=100, C=1, \Lambda=0.05$ and $0.62 \leq r_{0} \leq 0.83$. In all these examples we have $f_{-}\left(r_{0}\right)>0$ into the fixed intervals.

The other cases with $\Lambda>0(n \geq 10$ and $\alpha>0$ or $n \geq 5$ and $\alpha<0)$ we calculate the functions $\nu^{2}$ and $\kappa^{2}$ into a grid of points $\left(r_{0}, \Lambda, \tilde{\alpha}, n\right)$ and at each point of the grid these function are not positive at same time. The grid is built with $0.1 \leq r_{0} \leq 2$ (step 0.1$), 0 \leq \Lambda \leq 2$ (step 0.5), $5 \leq n \leq 20$ and $0.01 \leq \tilde{\alpha} \leq 100$ (step 0.01 into [0.01, 0.1], step 0.1 into $[0.1,1]$, step 1 into $[1,100])$ and $C=1$. In the case $\alpha<0$ we work with $-100 \leq \tilde{\alpha} \leq-0.01$, with the same steps for the corresponding intervals.

In these cases $(\Lambda>0)$, each value of $n$ has a different set of parameters $\left(r_{0}, \tilde{\alpha}, C\right)$ where both $\nu^{2}$ and $\kappa^{2}$ are positive, and this differences turns quit impossible to repeat the graphical analysis as in the $\Lambda<0$ cases. 

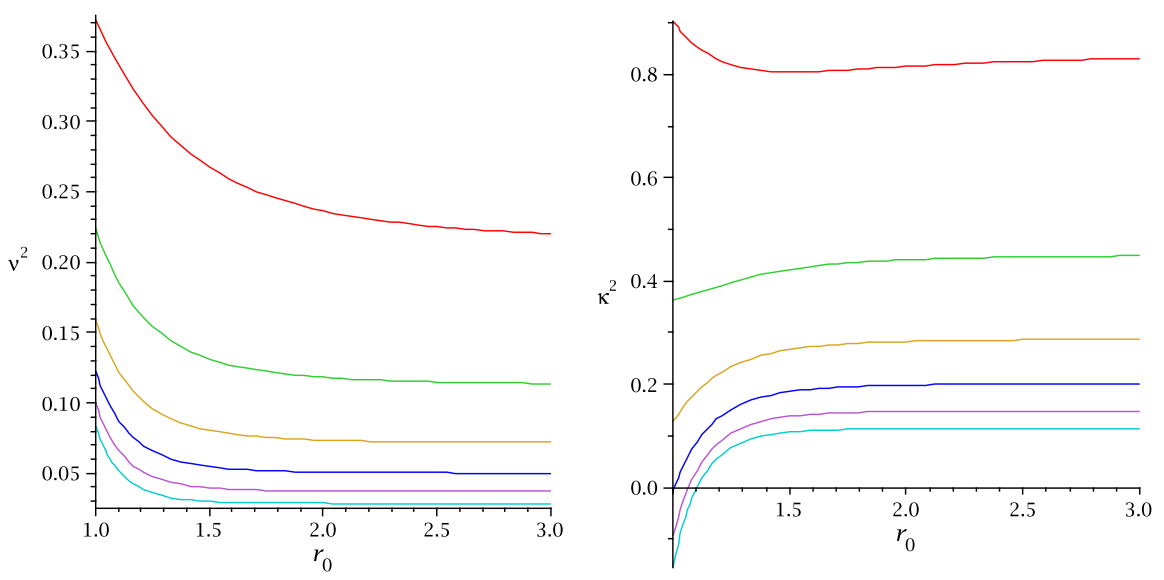

FIG. 3: GR branch, $\Lambda \neq 0$ case. The functions $\nu^{2}$ and $\kappa^{2}$ for $\Lambda=-1, C=1, \tilde{\alpha}=1$ and $1 \leq r_{0} \leq 3$, and $n=5$ (top curve), $6,7,8,9,10$ (bottom curve). For large $n$ we see a small region of instability.
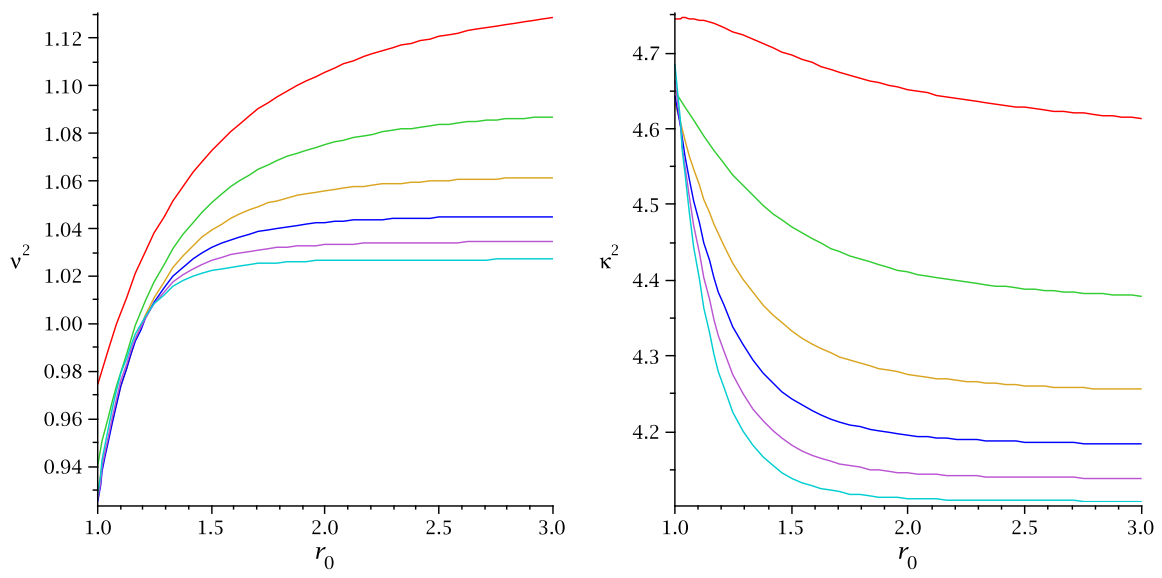

FIG. 4: Non-GR branch, $\Lambda \neq 0$ case. The frequencies $\nu^{2}$ and $\kappa^{2}$ for $\Lambda=1, C=1, \tilde{\alpha}=1,1 \leq r_{0} \leq 3$, and $n=5$,(top curve) $6,7,8,9,10$ (bottom curve). In this case we have stable orbits of arbitrary size.

\section{B. The non-GR branch solution $(\Lambda \neq 0)$}

Again, for completeness we describe the case where $f_{+}$is the solution of (40). In this case, the functions $\nu^{2}$ and $\kappa^{2}$ (26) are given as (45)) replacing $-\sqrt{\beta_{*}}$ by $\sqrt{\beta_{*}}$.

The condition to existence of $f\left(\beta_{*}>0\right)$ gives us $\left[(n-1)(n-2)+8 \Lambda \tilde{\alpha}+4 \tilde{\alpha} C r_{0}^{1-n}\right]>0$. For $\alpha<0$ the numerator of $\nu^{2}, N_{\nu}=r_{0}^{n}\left[(n-1)(n-2)\left(1+\sqrt{\beta_{*}}\right)+8 \Lambda \tilde{\alpha}-\tilde{\alpha} C r_{0}^{1-n}(n-5)\right]$, is always positive but the denominator, $D_{\nu}=\tilde{\alpha}(n-1)\left(+2 r_{0}^{n} \sqrt{\beta_{*}}(n-2)+r_{0}^{3} C\right)$, is negative, so $\nu^{2}<0$, and in this case no circular orbit is stable.

For $\alpha>0$ we first analyze the case $\Lambda>0$. In this case all parameters are positive and the denominators of $\nu^{2}$ and $\kappa^{2}$ are always positive. The same happens with $f_{+}, \beta_{*}, \tilde{B}_{4}, B_{3}, \tilde{B}_{2}$ and the expression $\left[(n-1)(n-2)\left(1+\sqrt{\beta_{*}}\right)+8 \Lambda \tilde{\alpha}\right]$ (Note that $\tilde{B}_{i}$ is the coefficient $B_{i}$ after replacing $-\sqrt{\beta_{*}}$ by $\sqrt{\beta_{*}}, i=2,3,4$ ). These positive coefficients assure that, for $r_{0}$ sufficiently large, the numerator of $\nu^{2}$ and $\kappa^{2}$ are positive. Hence, we conclude that there are stable circular orbits for $n \geq 5, \alpha>0$ and $\Lambda>0$.

If $\alpha>0$ and $\Lambda<0$, it is necessary to set conditions to assure $\beta_{*}>0$. It happens when $(n-1)(n-2)+8 \Lambda \tilde{\alpha}>0$, what is true when $2|\Lambda| \tilde{\alpha}<3$. In this case, from the expressions for $\nu^{2}$ and $\kappa^{2}$, we can see that the same argument used in the case $\Lambda>0$ is valid. Hence, we conclude that there are stable circular orbits for $n \geq 5, \alpha>0$ and $\Lambda<0$.

Figure (44) shows the functions $\nu^{2}$ and $\kappa^{2}$ for $\Lambda=1, C=1, \tilde{\alpha}=1,1 \leq r_{0} \leq 3$, and $n=5$, (top curve) $6,7,8,9,10$ (bottom curve). In this case we have stable orbits of arbitrary size.

We find a similar behavior for the equivalent case with $\Lambda=-1$. 


\begin{tabular}{|c|c|c|c|}
\hline Newtonian case & $n=4$ & $n=5$ & $n \geq 6$ \\
\hline$\Lambda=0$ & $\mathrm{SSC}$ & $\mathrm{NSC}$ & $\mathrm{NSC}$ \\
$\Lambda<0$ & $\mathrm{SSC}$ & $\mathrm{SSC}$ & $\mathrm{SSC}$ \\
$\Lambda>0$ & $\mathrm{SSC}$ & $\mathrm{NSC}$ & $\mathrm{NSC}$ \\
\hline
\end{tabular}

TABLE I: Stability of circular orbits in $n$-dimensional Newtonian gravity with or without cosmological constant. The existence of some stable curves is denoted by SSC, and their not existence by NSC.

\begin{tabular}{|c|c|c|}
\hline GR case & $n=4$ & $n \geq 5$ \\
\hline$\Lambda=0$ & $\mathrm{SSC}$ & $\mathrm{NSC}$ \\
$\Lambda<0$ & $\mathrm{SSC}$ & $\mathrm{SSC}$ \\
$\Lambda>0$ & $\mathrm{SSC}$ & $\mathrm{NSC}$ \\
\hline
\end{tabular}

TABLE II: Stability of circular orbits in n-dimensional Einstein gravity with or without cosmological constant. The existence of some stable curves is denoted by SSC, and their not existence by NSC.

\section{SUMMARY AND FINAL REMARKS}

An observationally viable theory of gravitation must allow for the existence of stable limited orbits. It is known that, in the Newtonian gravity as well as in classical general relativity, these orbits exist only if the dimension of the usual space is three $(n=4)$. We analyze the linear stability of circular geodesics in a $n$-dimensional $(n \geq 4)$ spacetime with not null cosmological constant $\Lambda$ and we find that there exist cases in which these orbits are stable.

We also analyse the linear stability of circular geodesics for $n$-dimensional spherically symmetric static solutions of Einstein-Gauss-Bonnet (EGB) gravity. Here we also find that there exist cases with stable circular orbits. The differential equation that gives us the metric function has two families of solutions corresponding to the sign in front of the square root in (29). Following the definition given at [20], the family with minus (plus) sign is called GR branch (non-GR branch) solution. The EGB part of our work is divided in several cases, depending on whether the cosmological constant $\Lambda$ is zero or not, and on the kind of solution used (GR or non-GR branch solution).

For the non-GR solution, the sign of the coupling parameter $\alpha$ is very important. When $\alpha<0$, no matter the value of $\Lambda$ or $n$, it is not possible to find stable circular orbits. Otherwise, when $\alpha>0$, for all $n \geq 5$, it is possible to find stable circular orbits, no matter whether $\Lambda$ is null, positive or negative.

The results obtained for the GR solution are similar. When $\Lambda$ is positive or null, and $\alpha$ is negative, the circular orbits are not stable. But we show values of $\alpha>0,5 \leq n \leq 9, \Lambda \geq 0$ and $r_{0}$ such that the existence of stable circular orbits is possible. The great difference of this branch solution occurs in the case $\Lambda<0$. In this case, for example, taking $\Lambda=-1, \alpha= \pm 1, n \geq 5$ and $r_{0} \geq 1.2$ we have stability. The results above summarized in tables (IIIII).

A similar analysis as the one presented in this article is under study for higher dimensions rotating black holes like the one presented in Refs. 22] (five-dimensional GR with magnetic field), 24] (n-dimensional GR) and 23] (five-dimensional EGB theory). Another possible generalizations and applications of the present work concerns with the study of gravitational radiation in higher dimensions in the case where circular orbits do exist. For gravitational radiation in $n$-dimensional spacetimes, see for instance 25]. Also the ultrarelativistic plunge into a multidimensional black hole was study in [26]. A generalization of this calculation to circular orbits may be relevant to improve our understanding of mini-black hole production in TeV-gravity scenarios.

\begin{tabular}{|c|c|c|c|c|c|c|}
\hline \multirow{2}{*}{$\begin{array}{c}\text { EGB } \\
(\alpha \neq 0)\end{array}$} & \multicolumn{2}{|c|}{$\Lambda=0$} & \multicolumn{2}{c|}{$\Lambda>0$} & \multicolumn{2}{c|}{$\Lambda<0$} \\
\cline { 2 - 7 } & $\alpha>0$ & $\alpha<0$ & $\alpha>0$ & $\alpha<0$ & $\alpha>0$ & $\alpha<0$ \\
\hline non-GR branch & SSC & NSC & SSC & NSC & SSC & NSC \\
\hline GR branch & SSC & NSC & SSC & NSC & SSC & SSC \\
\hline
\end{tabular}

TABLE III: Stability of circular orbits in Einstein-Gauss-Bonnet theory with or without cosmological constant and different signs for the EGB coupling constant. The existence of some stable curves is denoted by SSC, and their not existence by NSC. 


\section{Acknowledgments}

V.M.R. thanks the hospitality of DMA-IMECC-UNICAMP. P.S.L. thanks the partial financial support of FAPESP and CNPq. Also we thank Dr. J. Schleicher for his help with the English translation of the Kant's text.

[1] Free translation from: I. Kant, Gedanken von der wahren Schätzung der lebendigen Kräfte (1746). The original text can be found in http://www.philosophisches-lesen.de/kant/1746.html.

[2] For a discussion on Kant's dynamics see, M. Schönfeld, "Kant's early dynamics" in A companion to Kant, G. Bird, Ed. (Blackwell, Oxford 2006).

[3] E.J. Routh, A treatise on Dynamics of a Particle with Numerous Examples (Cambridge, 1898).

[4] P. Ehrenfest, Proc. Amsterdam Acad. 20, 200 (1917); Ann. Physik 61, 440 (1920).

[5] F.R. Tangherlini, Il Nuovo Cimento XXVII, 636 (1963).

[6] H. Bondi, Cosmology (Cambridge, 1960).

[7] D. Wilkins, Am. J. Phys. 54, 726 (1986).

[8] See for instance P.J.E. Peebles and B. Ratra, Rev. Mod. Phys. 75559 (2003).

[9] See for instance, M.P. do Carmo, Riemannian Geometry (Birkhäuser, Boston 1992).

[10] R. Bach, Math. Z. 9110 (1921).

[11] C. Lanczos, Z. Phys. 73147 (1932); Ann, Math. 39, 842 (1938).

[12] N. Deruelle and J. Madore, Arxiv:0305004 [gr-qc].

[13] D.G. Boulware and S. Deser, Phys. Rev. Lett. 552656 (1985)

[14] M.J. Duff, B.E.W. Nilsson, and C.N. Pope, Phys. Lett. B 173, 69 (1986).

[15] J. Binney and S. Tremaine, Galactic Dynamics (Princeton, 1987).

[16] D. Lovelock, J. Math. Phys. 12, 498 (1971)

[17] V. Fock, The Theory of Space, Time and Gravitation (Pergamon, London, 1964)

[18] G. Dotti and R.J. Gleiser, Phys. Rev. D 72, 044018 (2005).

[19] L. Abbott and S. Deser, Nucl. Phys. B 195, 1 (1982).

[20] M. Nozawa and H. Maeda, Class. Quantum Grav. 25055009 (2008).

[21] T. Torii and H. Maeda, Phys. Rev. D 71, 124002 (2005).

[22] A.N. Aliev V.P. and Frolov Phys. Rev. D 69, 084022 (2004).

[23] Y. Brihaye and E. Radu, arXiv:0801.1021.

[24] P. Krtous, D. Kubiznak, D. N. Page, M. Vasudevan, Phys. Rev. D 76084034 (2007).

[25] V. Cardoso, O.C. Dias, and J.P.S.Lemos Phys. Rev. D 67, 064026 (2003).

[26] E. Berti, M. Cavaglia, L. Gualtieri and Phys. Rev. D 69, 124011 (2004) 